\title{
Post-partum depression, anxiety and marital satisfaction: A perspective from Southeastern Nigeria
}

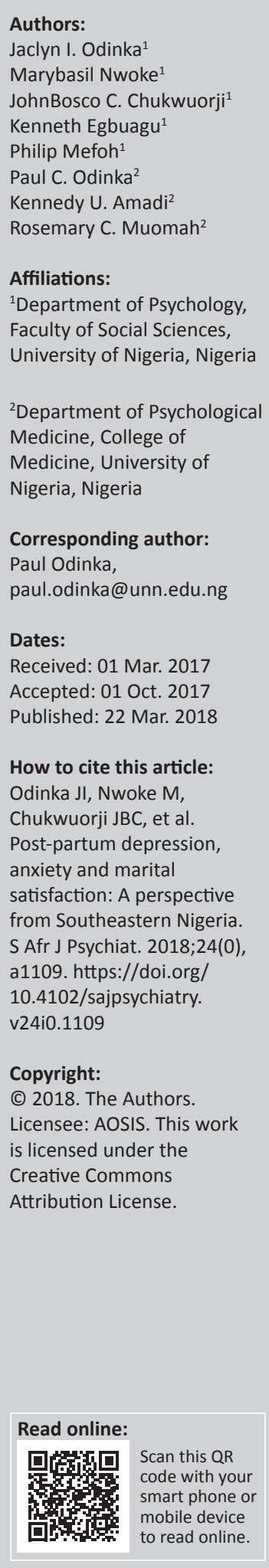

Background: Many studies have noted the high prevalence of post-partum depression (PPD) and anxiety associated with poor marital satisfaction, albeit amidst a dearth of literature on comorbid PPD and anxiety among women in Nigeria.

Objective: The study was aimed to assess the prevalence of PPD and anxiety, and to investigate their relationship with marital satisfaction in low-risk women in Enugu, Southeastern Nigeria.

Method: A cross-sectional study of 309 randomly selected nursing mothers at two tertiary health institutions. Socio-demographic Questionnaire, Hospital Anxiety and Depression Scale, and Index of Marital Satisfaction (IMS) were used to collect data on demography, anxiety and depression and marital relationship respectively. All statistical tests were performed at a significant level of 0.05 .

Results: The age range of the respondents was 20-46 years; mean and s.d. was 29.65 and \pm 4.87 , respectively, and most of them were graduates of tertiary educational institutions (74.1\%). The prevalence of post-partum anxiety was $31.1 \%$ and of PPD was $33.3 \%$. Marital dissatisfaction was observed in 39.5\% (122) of the respondents who were mothers. Those with co-morbid depression and anxiety $(22.0 \%)$ had worse marital dissatisfaction. The strongest correlation with depression and anxiety was item 12 of IMS ('feel that my partner doesn't confide in $\mathrm{me}^{\prime}$ ).

Conclusion: There was a high prevalence of marital dissatisfaction, PPD and anxiety among nursing mothers in Enugu, but with low detection rate. The effects of PPD and anxiety on the mother, her marital relationship and her infant make them essential conditions for early diagnosis, prevention and treatments.

\section{Introduction}

For most women having a baby, especially their first child, is a significant transitional life event. ${ }^{1}$ The first weeks and months postpartum may be associated with emotional upheaval. ${ }^{2}$ The nursing mother tries to meet the needs of her baby, to adapt to the changes in her sleep pattern and to recover from the process of delivery physically. ${ }^{3}$ Also, the depth of their feelings may be related to hormonal changes, fatigue and the pain of incisions, swollen breasts or sore nipples. ${ }^{4}$ Post-partum period involves changes in marital and family relationships and is commonly a cause of additional financial burden, even among households with relatively high income ${ }^{5}$ but worse among the low socioeconomic class. ${ }^{6,7}$

Post-partum depression (PPD) and anxiety affect approximately 10\% - 20\% of all mothers in Western societies. ${ }^{8}$ A prevalence of $23 \%$ - 31\% was reported for PPD in Southeastern Nigeria, 9,10 while Fatoye et al. ${ }^{11}$ reported that between $5 \%$ and $38 \%$ of women suffer from PPD in Southwestern Nigeria. The differences in the prevalence might be because of the effect of social, cultural, lifestyle and racial factors on depression. ${ }^{12}$ These statistics indicate that PPD might be a very common experience in Nigeria. Post-partum depression is related to the level of social support, ${ }^{2}$ previous depressive illness, stress, psychological factors and obstetric factors. ${ }^{6,7}$

Some psychosocial stressors in pregnancy may be because of cultural influence issues relevant to Igbo culture where marriage is patrilineal, with emphasis on begetting a child, especially a male child, to sustain a family lineage and to guarantee the position of the woman in the family. ${ }^{13}$ In Igbo culture and practices, not begetting a child, especially a male child, is one of the 
most relevant factors that could influence marital discord, and this could lead to marital dissatisfactions. Also, in Igbo culture and tradition, it is believed that a woman is not just married to a man but to the extended family, and for this, to a great extent, the in-laws have an influence on the marriage. Pressures from the extended family sometimes compound psychosocial stress postpartum. Psychosocial stressors in pregnancy or early postpartum is a strong predictor of postpartum depressive symptoms, ${ }^{14,15,16,17}$ and marital problems during pregnancy increase the risk of PPD and anxiety. ${ }^{18,19,20}$ However, the causal pathways between psychosocial stressors and depression may go in both directions. ${ }^{17}$

Post-partum depression can develop at any point during the first year of postpartum, with a peak of incidence in the first 4 months of postpartum. ${ }^{21,22}$ The mother may suffer an inability to take care of her new baby either emotionally or physically, which can impair early bonding and attachment process between mother and baby and could also hinder effective infant care skills ${ }^{1}$ and, if untreated, can affect the quality of mother-child relationship with deleterious effects on the child's cognitive, psychosocial and physical development. ${ }^{1,23}$ PPD may also adversely affect the marital relationship..$^{23}$

Depression often co-occurs with other disorders, especially anxiety symptoms..$^{24,25}$ Some authors ${ }^{26,27}$ have also found associations between experiencing stressful life events and maternal depression and anxiety. Previous studies have shown high correlations between measures of depressive and anxiety symptoms and relationship adjustment. ${ }^{25,28}$ Braverman and Britton ${ }^{29}$ reported a significant positive association between post-partum anxiety, as well as depression and infant mobility, temperament, adaptability and sensibility. Generally, PPD and anxiety may have implications for marital satisfaction.

Marital satisfaction can be defined as a subjective experience of one's personal happiness and contentment in a marital relationship. ${ }^{30}$ Greeff ${ }^{31}$ describes marital satisfaction as marital relationship in which couples have many agreements with each other on many variables such as family happiness, spouse's satisfaction with the sexual relationship, satisfaction with general quality of life, family strengths, flexibility in the way free time is spent in the marital relationship, sound relationships with family and friends, conflict management and resolution, and communication within the marriage. The couple is satisfied with the type and level of their relations and the type and quality of leisure times, and has proper management of their finances and time. ${ }^{31}$

There is a significant relationship among PPD, quality of marital relationship and mental health. ${ }^{32,33}$ Previous community surveys have shown that marital adjustment is concurrently, ${ }^{34}$ and prospectively, ${ }^{35}$ associated with anxiety disorders, and those anxiety symptoms could predict a decline in marital adjustment over time. ${ }^{36}$ Lower marital adjustment is prospectively associated with an onset of anxiety disorders in non-pregnant mothers. ${ }^{35}$
Given the high prevalence of PPD and anxiety, their multifactorial nature and their association with poor marital satisfaction, and the importance of mental health promotion in women, any attempt to reduce any risk factors affecting their incidence can lead to a decreased rate of depression and anxiety. The development of the relevant framework is important to develop the type of strategies that could be put in place for the prevention, early detection of women at risk, and for the appropriate intervention programme to be instituted for such mothers by addressing possible risk factors such as poor marital satisfaction through active case findings, counselling and social support during antenatal visits, as well as increasing the resources available for mental health services.

The study on PPD and anxiety becomes more imperative in this part of the world, and more so given the dearth of information on their correlates in the sub-Saharan African settings, when compared to the Western world, and the importance of mental health promotion in women. It is pertinent to conduct empirical investigations of factors associated with these disorders, as any reasonable attempt to reduce any of the confirmed risk factors affecting the incidence of PPD and anxiety can go a long way in reducing the high prevalence. This study was aimed to assess the prevalence of PPD and anxiety and to investigate their relationship with marital satisfaction in low-risk women in Enugu, Southeastern Nigeria. These should be an urgent challenge for Nigerian public health officials.

\section{Method Study area}

This study was carried out at the University of Nigeria Teaching Hospital (UNTH), Ituku-Ozalla, and Enugu State University of Science and Technology (ESUT) Teaching Hospital, both in Enugu, Enugu State, Southeastern Nigeria. The two hospitals, which are among the seven university teaching hospitals in Southeast Nigeria, provide medical services to residents of Enugu state and also receive referrals from and beyond all five southeastern states of Nigeria. Enugu is one of the most cosmopolitan Igbo cities and has the highest concentration of tertiary educational institutions in Southeastern Nigeria. Enugu is seen as a regional capital, as well as the Enugu State capital. Enugu State has a population of 3.3 million people of whom $95 \%$ are Igbo, and about $59 \%$ of the population live in the rural areas. ${ }^{37}$

Southeastern Nigeria (one of the six geopolitical zones or divisions in which Nigeria's 36 federating units or states are grouped) is primarily inhabited by the people of the Igbo ethnic group, who speak Igbo and one of the three largest and most influential ethnic groups in Nigeria. ${ }^{38}$ The Igbos constitute $18 \%$ of the total Nigerian population of 170 million, that is, approximately 30 million. ${ }^{39}$ Igbo people are predominantly Christians. ${ }^{38}$

\section{Sample identification and recruitment procedures}

Recruitment of participants was done at the postnatal clinics and Children's welfare clinics of the two hospitals. All the 
nursing mothers who came for follow-ups and immunisation for children, between November and December 2015, formed the sampling frame. With a prevalence of $23 \%{ }^{9}$ and an absolute standard error of 0.05 and a standard normal variance of 1.96, data collection was performed until the sample size of 309, considered adequate for the study, using the appropriate Cochran's sample size formula for proportions. ${ }^{40}$ With the help of the outpatients' attendance list, the mothers were randomly selected, using a table of random numbers. A total of 334 women were chosen for preliminary interviews to confirm their personal history, medical history, past psychiatric history, obstetric history and life events, and 309 who met the inclusion criteria were recruited into the study. The assessment was done from 6th week to 14th week of postpartum (the period from 2 nd to the 4th round of immunisation in Nigeria), as the highest prevalence has been observed within this time. ${ }^{22,41}$ Very minimal assistance was given to the mothers as they were literate enough to complete the questionnaires.

\section{Ethical issues}

First, the mothers were given a complete description of the study by one of the researchers, before a written informed consent was sought and obtained from them. The mothers who gave their consent were later given the three selfadministered questionnaires to complete.

They were told the average time it could take to complete the questionnaires and were given enough privacy. The questionnaires were anonymously completed, as they were also assured of confidentiality.

\section{Inclusion or exclusion criteria}

All the women that delivered their babies from the gestational age of 36 weeks were included in the study. Also, all women with conditions that might decrease the reliability of the instrument for the assessment of PPA and PPD, such as multiple births, obstetric and pregnancy complications, major chronic disease, any severe or unstable medical illness, a current or previous history of psychiatric disorders, and recent traumatic and life events, were excluded from the study. This information was through self-report during preliminary interviews.

\section{Instruments}

\section{Socio-demographic questionnaire}

Socio-demographic questionnaire was used to collect sociodemographic data such as age, the number of children, marital status, the area of residence (rural or urban), and educational attainment.

\section{Hospital Anxiety and Depression Scale}

Hospital Anxiety and Depression Scale (HADS) is a selfreport questionnaire ${ }^{42}$ and was developed to detect adverse anxiety and depressive states among outpatients in nonpsychiatric hospital clinics. Some of its positive qualities include brevity and easiness of use, excellent reliability and validity, as well as efficiency in screening and case-finding. ${ }^{43}$ It has also been found useful in the assessment of probable psychiatric morbidity in the community. Hospital Anxiety and Depression Scale consists of seven items each for depression and anxiety, and the scores are rated on a fourpoint scale ranging from 0 to 3 , with 21 as the highest score. For the stringent and robustness of case-findings, a cut-off point of 11 and above in either or both of the anxiety and depressive subscales indicates depression and anxiety disorder based on the DSM-IV and ICD-10 diagnostic criteria for current depressive disorder or anxiety disorder, while between 8 and 10 on both subscales indicates borderline case. It has been validated in many countries, among which is Nigeria. ${ }^{44}$

Hospital Anxiety and Depression Scale was not designed to be a clinical diagnostic tool but screening tool, ${ }^{45}$ and its use in this study was as a screening tool to flag probable depressive and anxiety illness and was usually a follow-up with a psychiatric interview in the psychiatric clinic for definitive diagnosis and treatment for women that needed such intervention. Therefore, its use in this study was to flag up probable depressive and anxiety illness and did not necessarily confer a diagnosing of depression and anxiety. However, HADS has also been found to perform well in assessing the severity in already diagnosed people with depression or anxiety. ${ }^{46}$

\section{Index of Marital Satisfaction}

Index of Marital Satisfaction (IMS) is a 25-item inventory, designed by Hudson, ${ }^{47}$ that measures the degree and severity or magnitude of the problems one spouse or partner perceives to be having in the marital relationship with his or her partner. The focus is on current problems that have reduced marital satisfaction. There is a direct scoring (items 2, 4, 6, 7, $10,12,14,15,18,22,24,25)$ and reverse scoring $(1,3,5,8,9,11$, $13,16,17,19,20,21,23)$ of the items. The final score is obtained by adding together the result of the direct scores and the reverse scores to obtain the client's raw score. The IMS was derived from subtracting 25 from the raw total score. A score below 30 was indicative of satisfaction with the relationship, while a score above 30 was indicative of dissatisfaction. Hudson ${ }^{47}$ reported a Cronbach's alpha of 0.96 and two-week intervals of test-retest reliability of 0.96 . It has been validated in many countries, and the internal consistency has been established in Nigeria. ${ }^{48}$

\section{Data analysis}

The raw data were keyed into Epidata software version 3.1 (The EpiData Association, Odense, Denmark) for data entry, documentation and storage, and was later transported to Statistical Package for the Social Sciences (SPSS) version 20.0 (IBM, USA) for analysis. The descriptive frequency distribution of the socio-demographic characteristics of the nursing mothers, the distribution of the mothers' anxiety and depression status and the distribution of the mothers' comorbidity for anxiety and depression were determined. 
Chi-square test was used to assess for an association between socio-demographic data and scores of IMS, and mothers with and without PPD and PPA, respectively. As scores on the IMS (continuous variables) did not have a Gaussian distribution, Spearman's correlations were performed to test the relationships between the Marital Satisfaction (independent variables) and the post-partum anxiety and depression (dependent variables). All statistical tests were two-sided and were performed at a significant level of 0.05 .

\section{Ethical consideration}

The approval for the study was sought and obtained from the institutional review board (IRB) of the College of Medicine, University of Nigeria Enugu Campus, Enugu, Nigeria. The study has, therefore, been performed in accordance with the ethical standards laid down in the 1964 Declaration of Helsinki and its later amendments.

\section{Results}

Table 1 shows the distribution of the mothers according to age range, marital status, educational status, the area of residence, employment status, religion, and ethnicity. The age range was 20-46 years; mean and S.D. were 29.65 and \pm 4.87 , respectively. Most of the respondents were graduates of tertiary educational institutions (74.1\%), and all the respondents went beyond the basic primary education with $1.6 \%$ of them dropping out of secondary school. About $97 \%$ of the mothers were married while $2.3 \%$ of them were single

TABLE 1: Distribution of mothers according to age range, marital status, educational status, area of residence, employment status, religion and ethnicity.

\begin{tabular}{|c|c|c|c|c|}
\hline Variables & $N$ & $\%$ & Mean & s.d. \\
\hline \multicolumn{5}{|l|}{ Age (years) } \\
\hline $20-25$ & 58 & 18.77 & 29.65 & \pm 4.87 \\
\hline $26-35$ & 221 & 71.52 & - & - \\
\hline $36-43$ & 25 & 8.09 & - & - \\
\hline $44-46$ & 5 & 1.62 & - & - \\
\hline \multicolumn{5}{|l|}{ Marital status } \\
\hline Married & 300 & 97.10 & - & - \\
\hline Divorced or separated & 2 & 0.60 & - & - \\
\hline Single & 7 & 2.30 & - & - \\
\hline \multicolumn{5}{|l|}{ Educational status } \\
\hline Secondary school completed & 30 & 9.70 & - & - \\
\hline Secondary school not completed & 5 & 1.60 & - & - \\
\hline Tertiary education completed & 229 & 74.10 & - & - \\
\hline Tertiary education not completed & 45 & 14.60 & - & - \\
\hline \multicolumn{5}{|l|}{ Area of residence } \\
\hline Urban area & 285 & 92.20 & - & - \\
\hline Rural area & 24 & 7.80 & - & - \\
\hline \multicolumn{5}{|l|}{ Employment status } \\
\hline Employed & 172 & 55.70 & - & - \\
\hline Unemployed or housewife & 81 & 26.20 & - & - \\
\hline Student & 56 & 18.10 & - & - \\
\hline \multicolumn{5}{|l|}{ Religion } \\
\hline Christianity & 308 & 99.70 & - & - \\
\hline Muslim & 1 & 0.30 & - & - \\
\hline \multicolumn{5}{|l|}{ Ethnicity } \\
\hline Igbo & 306 & 99.00 & - & - \\
\hline Others & 3 & 1.00 & - & - \\
\hline Total & 309 & 100 & - & - \\
\hline
\end{tabular}

parents. The majority of the mothers (55.7\%) were employed, while about $26 \%$ of them were unemployed.

The prevalence of post-partum anxiety was $31.1 \%$, while that of PPD was 33.3\%, and the prevalence of post-partum co-morbid anxiety and depression was $22 \%$ (See Table 2).

The scores on IMS scale were not normally distributed and had a median of 22.0. Note that the IMS scores above 39 indicate marital dissatisfaction, and $39.5 \%$ (122) of the mothers had marital dissatisfaction. The distribution of the scores of women on the IMS showed that 77 of $309(24.9 \%)$ of the women scored 42 and fell within the 75 th percentile. Four $(1.0 \%)$ of them had anxiety, $15(4.9 \%)$ had depression, and $33(10.7 \%)$ had both anxiety and depression while $25(8.1 \%)$ of them had neither anxiety nor depression. The numbers of women with both depression and anxiety had worse marital dissatisfaction, compared to those with either anxiety or depression alone.

Table 3 shows a Spearman's rank-order correlation coefficient, which was run to assess the relationship among PPD, postpartum anxiety and marital satisfaction. Preliminary analysis showed the relationship to be monotonic, as assessed by the visual inspection of a scatter plot. There was a moderate positive correlation between total score on IMS and HADS total score on Depression Subscale, $r^{s}=0.419, p<0.01$. There was also a moderate positive correlation between total score on IMS and total score on HADS (Anxiety Subscale), $r^{s}=$ $0.401, p<0.01$, where $r$ is the degree of freedom, obtained from the 'Sig. (two-tailed)' row. (Note: The higher the score on IMS, the more dissatisfaction with the relationship is indicated).

A chi-square test for an association was conducted to examine the differences in socio-demographic data between mothers with and without PPD and PPA using the dichotomised measures of both the dependent and independent variables. The chi-square analysis found that there was a statistically significant association between educational attainment and $\operatorname{PPD}\left(\chi^{2}[1]=7.797, p=0.005\right)$ and PPA $\left(\chi^{2}[1]=8.537, p=0.003\right)$.

A chi-square test for association was conducted examining the differences in socio-demographic data between mothers with and without marital satisfaction using the dichotomised measures of both the dependent and independent variables. The chi-square analysis found that there was a statistically significant association between age and marital satisfaction, $\left(\chi^{2}[1]=4.890, p=0.027\right)$. There was also a statistically significant association between educational attainment and marital satisfaction $\left(\chi^{2}[1]=13.978, p=0.005\right)$.

In Table 4, HADS anxiety subscale was positively related to most of the IMS items, except items 4 (I feel that I would not choose the same partner if I had to do it over), 10 (I feel that our life together is dull), 15 (I feel that we do not have enough interests in common) and 22 (I feel that we should do more things together). The strongest correlation with anxiety was 
TABLE 2: Distribution of respondents according to the severity of depression and anxiety, and comorbidity between post-partum depression and anxiety.

\begin{tabular}{lccccccc}
\hline Clinical status & Frequency & Percent & Mean & s.d. & Median & Mode \\
\hline Distribution of the mothers' anxiety status according to HADS & & & \\
Normal (0-7) & 148 & 47.9 & 8.39 & 3.914 & 8.00 & 7 \\
Mild (8-10) & 68 & 22.0 & - & - & - & - \\
Moderate (11-14) & 67 & 21.7 & - & - & - & - \\
Severe (15-21) & 26 & 8.4 & - & - & - & - \\
Distribution of the mothers' depression status according to HADS & & \\
Normal (0-7) & 142 & 46.0 & 8.44 & 3.869 & 8.00 & 7 \\
mild (8-10) & 64 & 20.7 & - & - & - & - \\
Moderate (11-14) & 85 & 27.5 & - & - & - & - \\
severe (15-21) & 18 & 5.8 & - & - & - & - \\
Distribution of the mothers' comorbidity for anxiety and depression & & \\
according to HADS & 76 & 24.6 & - & - & - & - \\
Normal (0-7) & 131 & 42.4 & - & - & - & \\
Mild -Severe (8-21) & 68 & 22.0 & - & - & - & - \\
Moderate -Severe (11-21) & 6 & 1.94 & - & - & - & - \\
Severe (15-21) & & &
\end{tabular}

s.d., standard deviation; HADS, Hospital Anxiety and Depression Scale.

TABLE 3: Spearman's rank-order correlation coefficient, assessing the relationship between post-partum depression, post-partum anxiety and marital satisfaction. Correlations IMS total score HADS total score HADS total score (depression (anxiety subscale) subscale)

\begin{tabular}{lccc}
\hline \multicolumn{3}{c}{} & \\
IMS total score & & & \\
Correlation coefficient & 1.000 & 309 & $0.401^{* *}$ \\
$N$ & 309 & & 309 \\
HADS total score (depression subscale) & 1.000 & $0.631^{* *}$ \\
Correlation coefficient & $0.419^{* *}$ & 309 & 309 \\
$N$ & 309 & & \\
HADS total score (anxiety subscale) & $0.401^{* *}$ & $0.631^{* *}$ & 1.000 \\
Correlation coefficient & 309 & 309 & 309 \\
$N$
\end{tabular}

IMS, Index of Marital Satisfaction; HADS, Hospital Anxiety and Depression Scale. $* p<0.05, * * p<0.01 ; * * * p<0.001$

found in item 12 (I feel that my partner doesn't confide in me) $(r=0.37, p<0.001)$.

The HADS Depression subscale was positively related to most of the IMS items, except items 3 (I feel that my partner really cares for me), 15 (I feel that we do not have enough interests in common) and 22 (I feel that we should do more things together). Items 4 and 22 which did not correlate significantly with anxiety were found to be significant correlates of depression. It was only item 22 that did not significantly correlate with any of the HAD subscales. The strongest correlation with depression was also found in item 12 (I feel that my partner does not confide in me) $(r=0.43, p<0.001)$.

\section{Discussion}

The study focused on women, who were in their 6th-14th weeks of postpartum, and was aimed to assess PPD and anxiety and to investigate their relationship with marital satisfaction in low-risk women. Over $74.0 \%$ of the respondents were graduates with a university degree, and all the respondents went beyond the basic primary education with only $1.6 \%$ of them dropping out of secondary school. This was a reflection of the realities of the Igbo society where high premium is, at present, placed
TABLE 4: Pearson's correlation coefficient, assessing the relationship between post-partum depression, post-partum anxiety, and 25 items of IMS.

\begin{tabular}{|c|c|c|c|}
\hline Number & IMS items & Anxiety & Depression \\
\hline 1 & I feel that my partner is affectionate enough & $0.29 * * *$ & $0.18^{* *}$ \\
\hline 2 & I feel that my partner treats me badly & $0.16 * *$ & $0.27 * * *$ \\
\hline 3 & I feel that my partner really cares for me. & $0.13^{*}$ & 0.02 \\
\hline 4 & $\begin{array}{l}\text { I feel that I would not choose the same partner } \\
\text { if I had to do it over. }\end{array}$ & 0.09 & $0.21 * * *$ \\
\hline 5 & I feel that I can trust my partner. & $0.26 * * *$ & $0.28 * * *$ \\
\hline 6 & I feel that our relationship is breaking up & $0.12 *$ & $0.19 * *$ \\
\hline 7 & I feel that my partner does not understand me. & $0.24 * * *$ & $0.40 * * *$ \\
\hline 8 & I feel that our relationship is a good one. & $0.25 * * *$ & $0.24 * * *$ \\
\hline 9 & I feel that ours is a very happy relationship & $0.27 * * *$ & $0.24 * * *$ \\
\hline 10 & I feel that our life together is dull & 0.10 & $0.12 *$ \\
\hline 11 & I feel that we have a lot of fun together. & $0.33 * * *$ & $0.25 * * *$ \\
\hline 12 & I feel that my partner doesn't confide in me & $0.37 * * *$ & $0.43 * * *$ \\
\hline 13 & I feel that ours is a very close relationship & $0.27 * * *$ & $0.20 * *$ \\
\hline 14 & I feel that I cannot rely on my partner & $0.26 * * *$ & $0.38 * * *$ \\
\hline 15 & $\begin{array}{l}\text { I feel that we do not have enough interests in } \\
\text { common }\end{array}$ & -0.00 & 0.08 \\
\hline 16 & $\begin{array}{l}\text { I feel that we manage arguments and } \\
\text { disagreements very well }\end{array}$ & $0.23 * * *$ & $0.26 * * *$ \\
\hline 17 & $\begin{array}{l}\text { I feel that we do a good job of managing our } \\
\text { finances }\end{array}$ & $0.22 * * *$ & $0.17 * *$ \\
\hline 18 & $\begin{array}{l}\text { I feel that I should never have married my } \\
\text { partner }\end{array}$ & $0.14 *$ & $0.14 *$ \\
\hline 19 & $\begin{array}{l}\text { I feel that my partner and I get along very well } \\
\text { together }\end{array}$ & $0.25 * * *$ & $0.29 * * *$ \\
\hline 20 & I feel that our relationship is very stable & $0.26 * * *$ & $0.25 * * *$ \\
\hline 21 & $\begin{array}{l}\text { I feel that my partner is pleased with me as a } \\
\text { sex partner }\end{array}$ & $0.17 * *$ & $0.12 *$ \\
\hline 22 & I feel that we should do more things together & -0.01 & 0.06 \\
\hline 23 & $\begin{array}{l}\text { I feel that the future looks bright for our } \\
\text { relationship }\end{array}$ & $0.24 * * *$ & $0.22 * * *$ \\
\hline 24 & I feel that our relationship is empty & $0.17 * *$ & $0.29 * * *$ \\
\hline 25 & I feel there is no excitement in our relationship & $0.18 * *$ & $0.21 * * *$ \\
\hline
\end{tabular}

IMS, Index of Marital Satisfaction.

${ }^{*} p<0.05, * * p<0.01 ; * * *<0.001$

on education, including, among the female gender, which is in line with the aphorism that said that when you educate a man, you educate a person, but when you educate a woman, you educate a complete nation; as the education of her children would be highly guaranteed; moreover, the education of every child starts from the family, and the mother is the first teacher. ${ }^{49}$

The majority of the mothers $(55.7 \%)$ were employed, while the unemployed or housewives and students constituted $26.0 \%$ and $17.2 \%$, respectively. As such, beyond their indispensable roles as mothers and wives, women have remarkably contributed to the building of their nation's economy as skilled and unskilled labourers, artisans, traders, farmers, civil servants, and professionals in different areas of human endeavour. ${ }^{50}$ With these responsibilities, the nursing mother tries to meet the needs of her new born to adapt to the changes in her sleep pattern (most often the lack of sleep) and to recover physically from the delivery. ${ }^{4}$ All these factors could put the new mother at risk for psychological distress during the post-partum period. ${ }^{5}$

Less than $3 \%$ of the mothers were single parents; this could be related to the cultural factors primarily, as Igbo culture believes so much in strong familial ties and does not encourage single parenthood in a mother's parental home. 
This aspect of Igbo culture is taken seriously, given the shame it brings to a single mother and her family, fears of parental rejection or violent reaction. ${ }^{51}$ In addition, there is fear of stigmatisation and discrimination from her peers and family members and friends, partner's negative reaction or refusal to admit being responsible for the pregnancy, poor financial support, religious ostracisation, social isolation, abuse, and expulsion from school or work. ${ }^{52,53}$ Some factors could combine to make it very unlikely for many of such single teenage mothers to visit postnatal clinics in a tertiary hospital and could also force many of them into sheltered homes where they are taken care of, right from pregnancy to puerperium. However, Ossai and Uzochukwu, ${ }^{54}$ in their study, reported a higher rate (urban area $=7.8 \%$ and rural area $=10.7 \%$ ) in a survey of 540 clients of maternal health service in 18 of 440 primary health care centres in urban and rural primary health care centres in Enugu state, Southeast Nigeria, who attended antenatal and postnatal clinics.

Results showed that a considerable subgroup of nursing mothers $(33.3 \%)$ reported moderate-to-severe symptoms of depression; while the mothers with some possibility of having PPD (mild depression) constituted $20.7 \%$ of the mothers. Those with severe depression were $5.8 \%$. The mean score of women's PPD was $8.44 \pm 5$. 3.87. Previous research in PPD has recorded a prevalence rate of between $5.0 \%$ and $38.0 \% .8,9,10,11$

Also, $35.9 \%$ of the mothers reported moderate-to-severe symptoms of anxiety; $22.0 \%$ of the mothers had mild PPA. Those with severe anxiety were $8.4 \%$. The mean score of women's post-partum anxiety was $8.39 \pm 3.91$. Previous research in PPA has recorded a prevalence rate of between $10.0 \%$ and $20.0 \%{ }^{8}{ }^{811}$ The differences in the prevalence might be because of the effect of social, cultural, lifestyle and racial factors on depression and anxiety. ${ }^{12}$

The prevalence of co-morbid post-partum anxiety and depression was found to be $22.0 \%$; previous studies had reported prevalence ranging from $2.0 \%$ to $13.0 \%$ of comorbidity for PPD and anxiety in the first year. ${ }^{55,56}$

The study observed that the numbers of women with both depression and anxiety had worse marital dissatisfaction, compared to those with either anxiety or depression alone, and this is in line with report of previous studies suggesting that the mental health benefits of marriage are greater for those in 'good-quality' relationships in comparison to those in 'poor-quality' relationships. ${ }^{57}$

The study found an association between educational attainment and PPD and PPA. Previous studies have pointed out the role of educational attainment in the aetiology of psychological distress, and low education has been pointed out as a risk factor in mental health disorders. ${ }^{58}$

The study also found an association between marital satisfaction and age, and this was supported by previous studies. ${ }^{59}$ Also, there was an association between marital satisfaction and education attainment, and this is supported by the previous study. ${ }^{60}$

More importantly, this study showed that there is a significant and inverse relationship between marital satisfaction and post-partum anxiety, and this is supported by previous research involving community samples, which has shown that poor marital adjustment is associated with anxiety disorders. ${ }^{34,35}$ Also, in this the study, a moderate and inverse relationship between marital satisfaction and PPD was observed, and this has been reported in related previous studies elsewhere. ${ }^{32,61,62}$ However, $\mathrm{O}^{\prime}$ Hara and Swain ${ }^{14}$ reported a small inverse relationship between poor marital relationship and PPD. Most women with PPD have lower levels of marital satisfaction and higher stress, and this may indicate the protective role of marital satisfaction in $\mathrm{PPD}^{32}$ and vice versa. The causal pathways between psychosocial stressors (as in degree of marital satisfaction) and depression may go in both directions. ${ }^{17}$ Invariably, the study showed that there was a significant inverse correlation between depression, and anxiety with marital satisfaction in the post-partum period, and this has been reported in previous studies. 2,26,27,63 Atkinson and Rickel ${ }^{64}$ found that, consistent with the behavioural perspective of PPD, perceived positive reinforcement was inversely related to PPD. The lack of intimacy and marital strife is linked to depression in women, ${ }^{65}$ and anxiety symptoms predict a decline in marital adjustment over time. ${ }^{36}$

The study found out in the analysis that item 12, which said: 'I feel that my partner doesn't confide in me', had the strongest correlation with anxiety and depression. In a systematic review of 20 studies carried out in both rural and urban parts of Pakistan, ${ }^{66}$ reported the absence of a confiding relationship, as among the other four factors perceived by women to affect their mental health. ${ }^{66}$ This may not be out of place in Igbo culture, where the women have placed high premium on the trust of their husbands, ${ }^{67}$ and the husbands not confiding in their wife would most likely be perceived by a woman as because of the lack of trust and confidence in her. The perceived lack of trust and confidence could be a source of significant psychosocial distress for the women, and this could serve as a guide to areas that can be targeted in preventative programs, that will also involve couple therapy. The quality of marital relationship significantly affects the quality of mental health, ${ }^{33}$ and psychologically distressed individuals inadvertently contribute to the occurrence of stress in their lives, including stress in their intimate relationships, which increases the probability of the onset and maintenance of psychological distress. ${ }^{68}$

\section{Limitations of the study}

The limitations of this study include the cross-sectional design (the study design should stand out clearly in the methodology), which limited the ability to draw causal conclusions. Also, this study relied exclusively on selfreported on marital satisfaction, symptoms of depression and anxiety; as such risk of inaccurate reporting may exist especially as no formal diagnostic interview was carried out. 
The problems that could have been associated with the pregnancy, which might have increased the psychosocial stressors, and contributions to the amount of marital disharmony experienced, were not recorded.

The researcher did not investigate the role of biological factors such as hormonal changes, psychological factors such as personality and indeed other possible factors such as substance use in determining depression and anxiety. Also, there was no adequate investigation on whether marital satisfaction caused depression and anxiety or whether depression and anxiety caused marital satisfaction, as the causal pathways may go in both directions. The inadvertent inclusion of the single mothers in the analysis might have to a slight extent, influenced the outcome of the study. As the study was conducted in two tertiary hospital populations of PPD and anxiety, it is possible that mothers with more problematic or complicated pregnancies were more than represented in the studied population. All these should be identified as significant limitations in generalising the findings, and these limitations need to be considered in future.

\section{Conclusion}

There was a high prevalence of marital dissatisfaction, PPD and anxiety among nursing mothers in Enugu but with low detection rate. The effects of PPD and anxiety on the mother, her marital relationship and her infant make them essential conditions for early diagnosis, prevention and treatments.

\section{Acknowledgements Competing interests}

The authors declare that they have no financial or personal relationships which may have inappropriately influenced them in writing this article.

\section{Authors' contributions}

All authors contributed to the conception of the study. J.I.O. wrote the initial draft of the manuscript. J.I.O., M.N., J.C.C., K.E., P.M., P.C.O., K.U.A. and R.C.M. were involved in revising the manuscript. All authors read and approved the final draft of the manuscript. The requirements for authorship as demanded by this journal have been met, and each author believes that the manuscript represents honest work.

\section{References}

1. Yelland J, Sutherland G, Brown SJ. Postpartum anxiety depression and social health: Findings from a population-based survey of Australian women. BMC Public Health. 2010;10:771. https://doi.org/10.1186/1471-2458-10-771

2. Newton-Wellesley Hospital. Postpartum emotional adjustments [homepage on the Internet].[cited 2016 Dec 10]. Available from: www.nwh.org/communityhealth-resources/postpartum-guide/care-of-yourself/emotional-adjustments/

3. Hilli R. Evidence-based public health practices for screening for postpartum depression [homepage on the Internet]. [cited 2016 Nov 28]. Available from: http://corescholarlibrarieswrightedu/mph/48

4. Pelv-lce. Postpartum skin changes. In: Naturally supporting the way you heal postpartum [homepage on the Internet]. [cited 2016 Nov 28]. Available from: http://www.mamastrut.com/knowledge_center/page/2/

5. Braveman P, Marchi K, Kim S, et al. Poverty near poverty and hardship around the time of pregnancy. Matern Child Health J. 2010;14:20-35.https://doi. org/10.1007/s10995-008-0427-0
6. Di Marco S. Gambaro E, Zeppegno P, et al. 1755 - Postpartum depression anxiety and marital relationship: A pilot study. Eur Psychiatry. 2013:28(1):1. https://doi. org/10.1016/S0924-9338(13)76733-6

7. Robertson $E$, Grace $S$, Wallington $T$, et al. Antenatal risk factors for postpartum depression: A synthesis of recent literature. Gen Hosp Psychiatry. 2004;26: 289-295. https://doi.org/10.1016/j.genhosppsych.2004.02.006

8. O'Hara MW, McCabe JE. Postpartum depression: Current status and future directions. Ann Rev Clin Psychol. 2013;9:379-407. https://doi.org/10.1146/ annurev-clinpsy-050212-185612

9. Chinawa JM, Odetunde OI, Ndu IK, et al. Postpartum depression among mothers as seen in hospitals in Enugu South-East Nigeria: An undocumented issue. Pan Afr Med J. 2016;23:180. https://doi.org/1011604/pamj2016231808244

10. Ukaegbe $\mathrm{Cl}$, Iteke $\mathrm{OC}$, Bakare $\mathrm{MO}$, et al. Postpartum depression among Igbo women in an Urban Mission Hospital South East Nigeria. EMJ. 2012;11(1\&2):29-36.

11. Fatoye FO, Oladimeji BY, Adeyemi AB. Difficult delivery and some selected factors as predictors of early postpartum psychological symptoms among Nigerian women. J Psychosom Res. 2006;60:299-301. https://doi.org/10.1016/j. jpsychores.2005.04.012

12. Bakhshayesh A, Mortazavi M. The relationship between sexual satisfaction general health and marital satisfaction in couples. J Appl Psychol. 2011;4(12):73-85.

13. Obi CA. Marriage among the Igbo of Nigeria. ATR Special Topics. 1970. Available from: http://nigeriamasterweb.com/igbomarriage.html

14. O'Hara MW, Swain AM. Rates and risk of postpartum depression: A meta-analysis. Int Rev Psychiatry. 1996;8:37-54. https://doi.org/10.3109/09540269609037816

15. O'Hara MW, Schlechte J, Lewis D, et al. Controlled prospective study of postpartum mood disorders: Psychological environmental and hormonal variables. Psychol. 1991;100:63-73. https://doi.org/10.1037/0021-843X.100.1.63

16. Spangenberg JJ, Pieters HC. Factors related to postpartum depression. S Afr Psychol. 1991;1(3):159-165. https://doi.org/10.1177/008124639102100305

17. House JD, Iriarte RI, Burns EA. Stressful life events and depressive symptomatology in obstetric patients: A pilot study in a family practice setting. Fam Pract Res J. 1986;2(2):98-105.

18. Akbarzadeh M, Sharif F, Zare N, et al. Prevalence of symptoms post-partum anxiety and baby blues and factors effective upon it in women with high risk pregnancies. J Fam Res. 2009;5(17):57-71.

19. Braverman J, Roux JF. Screening for the patient at risk for postpartum depression. Obstet Gynecol. 1978;52:731-736.

20. Kumar R, Robson KM. A prospective study of emotional disorders in childbearing women. Br J Psychiatry. 1984;144:35-47. https://doi.org/10.1192/bjp.144.1.35

21. McCoy SJB, Beal JM, Shipman SB, et al. Risk factors for postpartum depression A retrospective investigation at 4-weeks postpartum and a review of the literature. J Am Osteopath Assoc. 2006;106:193-198.

22. O'Hara MW, Zekoski EM, Phillips LH, et al. A controlled prospective study of postpartum mood disorders: Comparison of childbearing and non-childbearing women. J Abnorm Psychol. 1990;99:3-15. https://doi.org/10.1037/0021-843X. 99.1 .3

23. Murray L, Halligan SL, Cooper PJ. Effects of postnatal depression on mother-infant interactions and child development. In: Wachs T, Bremner G, editors. Handbook of infant development. Oxford: Wiley-Blackwell, 2009; p. 192-220.

24. Kessler RC, Berglund $P$, Demler $O$, et al. The epidemiology of major depressive disorder: Results from the National Co-morbidity Survey Replication (NCS-R). J Am Med Assoc. 2003;289:3095-3105.https://doi.org/101001/jama28923 3095

25. Watson D. Differentiating the mood and anxiety disorders: A quadripartite model. Ann Rev Clin Psychol. 2009;5:221-247.https://doi.org/101146/annurevclinpsy 032408153510

26. Johnstone S, Boyce P, Hickey A, et al. Obstetric risk factors for postnatal depression in urban and community samples. AustN Z J Psychiatry. 2001;35:69-74. https:// doi.org/10.1046/j.1440-1614.2001.00862.x

27. Herrick $H$. The effect of stressful life events on postpartum depression: Results from the 1997-1998 North Carolina Pregnancy Risk Assessment Monitoring System (PRAMS) [homepage on the Internet].SCHS Students No.121.A Special Report Series by the State Center for Health Statistics. 2000; p. 1-9. Available from: http://citeseerx.ist.psu.edu/viewdoc/summary?doi=10.1.1.177.1300

28. Whisman MA, Beach SRH. Models for understanding interpersonal processes and relationships in anxiety disorders. In: Beck JG, editor. Interpersonal processes in the anxiety disorders: Implications for understanding psychopathology and treatment. Washington, DC: American Psychological Association, 2010; p. 9-35.

29. Braverman R, Britton JR. Infant temperament and maternal anxiety and depressed mood in the early postpartum period. Women Health. 2011;51(1):55-71. https:// doi.org/10.1080/03630242.2011.540741

30. Hendrick SS, Hendrick C. Love and satisfaction. In: Strenberg RJ, Hojjat M, editors Satisfaction in close relationships. New York: Guildford Press, 1997; p. 56-78.

31. Greeff AP. Characteristics of families that function well. J Fam Issues. 2000;21(8):948-962. https://doi.org/10.1177/019251300021008001

32. Khadivzadeh T, Kiani F, Sargolzaee M, et al. Examining the relationship between marital satisfaction three months after pregnancy and postpartum depression Global Conference on Health Promotion: The role of nurses and midwives. Hamedan, Iran: Hamedan University: School of Nursing and Midwifery University of Medical Sciences and Health Services;2009.

33. Hadizadeh TF, Bahri N, Tavakolizad J. Comparison of postpartum depression in normal and emergency cesarean section in primiparous women visited 22 Bahman hospital Gonabad City. J Kermanshah UnivMed Sci. 2003;4:21-30. 
34. Whisman MA. Marital distress and DSM-IV psychiatric disorders in a populationbased national survey, I Abnorm Psychol. 2007:116:638-643 https://doi. org/10.1037/0021-843X.116.3.638

35. Overbeek G, Vollebergh W, de Graaf R, et al. Longitudinal associations of marital quality and marital dissolution with the incidence of DSM-III-R disorders. J Fam Psychol. 2006;20:284-291.https://doi.org/10.1037/0893-3200.20.2.284

36. Dehle C, Weiss RL. Associations between anxiety and marital adjustment. J Psychol. 2002;136:328-338 https://doi.org/101080/00223980209604160

37. Makama SID. Report of Nigeria's National Population Commission on the 2006 census. Popul Dev Rev.2007;1(33):206-210.

38. Odinka PC, Ndukuba AC, Muomah RC, et al. Positive and negative symptoms of schizophrenia as correlates of help-seeking behaviour and the duration of untreated psychosis in south-east Nigeria. S Afr J Psychiatry.2014;20(4):166-171. https://doi.org/107196/SAJP536

39. Central Intelligence Agency. Calculation from percentage and overall population count of Nigeria. In: CIA, editor. The world factbook. Langley, VA: Directorate of count of Nigeria. In:
Intelligence, 2015.

40. Cochran WG. Sampling techniques. 3rd ed. New York: Wiley; 1977.

41. Miller RL, Pallant JF, Negri LM. Anxiety and stress in the postpartum: Is there more to postpartum distress than depression? BMC Psychiatry. 2006;6:12. https://doi. org/10.1186/1471-244X-6-12

42. Zigmond AS, Snaith RP. The hospital anxiety and depression scale. ActaPsychiatrScand. 1983;67:361-370. https://doi.org/10.1111/j.1600-0447.1983 tb09716.x

43. Luckett T, Butow PN, King MT, et al. A review and recommendations for optimal outcome measures of anxiety depression and general distress in studies evaluating psychosocial interventions for English-speaking adults with heterogeneous cancer diagnoses. Support Care Cancer. 2010;18:1241-1262.https://doi.org/101007/ s00520-010-0932-8

44. Abiodun OA. A validity study of the Hospital Anxiety and Depression Scale in general hospital units and a community sample in Nigeria. Br J Psychiatry. 1994;165(5):669-672. https://doi.org/10.1192/bjp.165.5.669

45. Whelan-Goodinson R, Ponsford J, Schönberger M. Validity of the Hospital Anxiety and Depression Scale to assess depression and anxiety following traumatic brain injury as compared with the structured clinical interview for DSM-IV. J Affect Disord. 2009;114(1):94-102. https://doi.org/10.1016/j.jad.2008.06.007

46. Bjelland I, Dahl AA, Haug TT, Neckelmann D. The validity of the Hospital Anxiety and Depression Scale; an updated review. J Psychiat Res. 2002;52:69-77. https:// doi.org/10.1016/S0022-3999(01)00296-3

47. Hudson WW. Index of marital satisfaction: The clinical measurement package: A field manual. Chicago, IL: Dersey Press; 1982.

48. Kolo FD. Sex religious background and educational qualification variables on sexual dissatisfaction among couples. IFE Psychol. 1994;2(2):118-128.

49. Amaghiro OC. Girl-child education a sustainable strategy for Igbo development. An abstract from the 13th International Conference of the Igbo Studies Association Conference;2015 April 9-11. Marquette, WI: Center Marquette University;2015.

50. Amajuoyi UF. 'OganihuUmunwanyi': The perspectives of women's commercial synergy. An abstract from the 13th International Conference of the Igbo Studies Association Conference;2015 April 9-11. Marquette, WI: Center Marquette University; 2015.

51. Claman AD, Williams BJ, Wogan $L$, et al. Reaction of unmarried girls to pregnancy. Can Med Assoc J. 1969;101:328-334.
52. Ilika A, Igwegbe A. Unintended pregnancy among unmarried adolescents and young women in Anambra State, south-east Nigeria. Afr J Reprod Health. 2004;8(3):92-102. https://doi.org/10.2307/3583396

53. Constance MU, Vaughn IR, Abbey BB. Are pregnant adolescents stigmatized by pregnancy? J Adolesc Health. 2004;36(4):352.e1-e8.https://doi.org/10.1016/j. jadohealth.2004.06.006

54. Ossai EN, Uzochukwu BS. Knowledge of danger signs of pregnancy among clients of maternal health service in urban and rural primary health centres of southeast Nigeria. J Community Med Health Educ.2015;5:337. https://doi.org/10.4172/ 2161-0711.1000337

55. Reck C, Struben K, Backenstrass M, et al. Prevalence onset and co-morbidity of postpartum anxiety and depressive disorders ActaPsychiatrScand. 2008;118: 459-468. https://doi.org/101111/j1600-0447200801264x

56. Austin MP, Hadzi-Pavlovic D, Priest SR, et al. Depressive and anxiety disorders in the postpartum period: How prevalent are they and can we improve their detection? Arch Women's Mental Health. 2010;13(5):395-401. https://doi. 10.1111/j.1600-0447.2008.01264.x

57. Leach LS, Butterworth P, Olesen SC, Mackinnon A. Relationship quality and levels of depression and anxiety in a large population-based survey. Soc Psychiatry PsychiatrEpidemiol. 2013;48(3):417-425. https://doi.org/10.1007/s00127-0120559-9

58. Patel V, Araya R, de Lima M, Ludermir A, Todd C. Women, poverty and common mental disorders in four restructuring societies. SocSciMed. 1999;49(11): 1461-1471. https://doi.org/10.1016/S0277-9536(99)00208-7

59. Dabone KT. Marital satisfaction among married people in Sunyani municipality[Doctoral dissertation]. Cape Coast: University of Cape Coast;2012.

60. Rachel M, Mirecki JL, Chou ME, Christine MS. What factors influence marital satisfaction? Differences between first and second marriages. J Divorce Remarriage. 2013;54(1):78-93. https://doi.org/10.1080/10502556.201 2.743831

61. Mobarak Abadi A, Fallahchai R, Mahin A. The relationship between marital satisfaction and postpartum depression in women who visited health centers in Bandar Abbas city. J Appl Environ Biol Sci. 2014;4(3):120-124.

62. Meçe D. Postpartum depression and marital relationship. Acad J Interdiscip Stud. 2013;2(4):319-325.

63. Whisman MA. Davila J, Goodman SH. Relationship adjustment depression and anxiety during pregnancy and the postpartum period. J Fam Psychol. 2011;25(3):375-383. https://doi.org/10.1037/a0023790

64. Atkinson AK, Rickel AU. Postpartum depression in primiparous parents. J Abnorm Psychol. 1984;93:115-119. https://doi.org/10.1037/0021-843X.93.1.115

65. Murphy MJ, Cowan RL. Psychiatry.4th ed. New York: Lippincott Williams and Wikkins; 2007.

66. Mirza I, Jenkins R. Risk factors, prevalence, and treatment of anxiety and depressive disorders in Pakistan: Systematic review. BMJ. 2004;328(7443):794. https://doi.org/10.1136/bmj.328.7443.794

67. Odimegwu C, Okemgbo CN. Gender role ideologies and prevalence of violence against women in Imo State, Nigeria. Anthropologist. 2003;5(4):225-236. https:// doi.org/10.1080/09720073.2003.11890814

68. Davila J, Bradbury TN, Cohan $\mathrm{CL}$, et al. Marital functioning and depressive symptoms: Evidence for a stress generation model. I PersSoc Psychol. 1997;73:849-861. https://doi.org/101037//0022-3514734849 